\title{
Applied research of RFID middleware system based on hierarchical structure
}

\author{
Zhengxi Wei \\ School of Computer Science, Sichuan University of Science \& Engineering, Zigong Sichuan 643000, PR China
}

Email address:

413789256@qq.com

To cite this article:

Zhengxi Wei. Applied Research of RFID Middleware System Based on Hierarchical Structure. Automation, Control and Intelligent Systems. Vol. 2, No. 6, 2014, pp. 112-116. doi: 10.11648/j.acis.20140206.13

\begin{abstract}
RFID middleware system, as the nerve center of many RFID systems, is being more and more concerned on by the people. It plays a key role in the development of the entire RFID industry. Firstly, this paper briefly introduces the present situation of RFID middleware, and then focuses on data filtering, data aggregation and information transmission three important technologies. According to the function demand, the next section introduces the hierarchical design idea for RFID middleware system. Based on the layered design idea, we construct a hierarchy structure of RFID middleware and present a kind of enterprise class applied model, in which RFID event manager module is responsible for processing data streams and RFID information service module is responsible for system integration. This applied model is easy to build a new RFID middleware system provides a kind of reference. Simulation and practical tests prove that the middleware system can real-time reads the important information of tracked objects such as goods in storehouse, achieve a more efficient management and facilitate practical application for Internet of things technology.
\end{abstract}

Keywords: RFID Middleware System, Structure Design, Applied model, Data Processing, Middleware Application

\section{Introduction}

Being contributed to Internet of things technology, a high degree of information sharing enables enterprises to optimize business processes and resource allocation, strengthening detail management and process management, promotes that enterprises have to continuously adapt to changes in the external environment, and improves the core competitiveness and innovation ability. For example, Lockheed Martin military company, which uses advanced management information system (MIS), during the development of JSF aircraft, tooling reduces $90 \%$, production time reduces $66 \%$ and manufacturing costs reduce $50 \%$.

With the rapid development of Internet of Things, RFID middleware technology [1] is integrated with other information technology, forming the core technology during the informatization procedure of manufacturing and services industry, driving its informatization extent to a new level, and promoting its sustainable development. RFID technology can be used to significantly improve the efficiency of business processes by providing the capability of automatic identification and data capture. This technology poses many new challenges on current data management systems. RFID data are time-dependent, dynamically changing, in large volumes, and carry implicit semantics. RFID data management systems need to support effectively such large-scale temporal data created by RFID applications. These systems need to have an explicit temporal data model for RFID data to support tracking and monitoring queries [2]. In addition, they need to have an automatic method to transform the primitive observations from RFID readers into derived data used in RFID-enabled applications.

RFID middleware is a kind of intermediate program, which achieves data transmission between the RFID hardware devices and software applications, filtering and converting data format. It reads the data from readers through the middleware extraction, decryption, filtered as well as format conversion, importing into enterprise information system to apply. Thus, it solves the problem that enterprises adopt RFID technology in the most time-consuming, labor-intensive highest complexity and difficulty, to ensure the reader to read the data correctly and import the data enterprise into the information systems according to the needs. In addition, RFID as a the key technology is also used in the field of 
manufacturing, assembly, postal services, warehousing and asset management, to build a smart RFID middleware system will make these applications become more convenient and efficient. In this paper, we present an integrated RFID middleware system based on hierarchical design idea and enterprise class applied model. Our system enables redundant RFID data filtering and automatic data transformation based on specific rules, provides powerful query support of RFID object tracking and management, and can be used to different RFID applications.

\section{Important Functions}

RFID middleware process data streams by distributed, hierarchical approach, with data collection, filtering, integration, delivery and other functions, so it is able to send information to the enterprise back-end applications, or other information systems. Thus, the following issues may occur in the course of information exchange. RFID device reading the data does not necessarily only use a particular application, so it may use multiple applications. Each application may also need many different sets of data, require data corresponding processing (such as redundant data filtering, etc.). This article next focuses on three key issues: data filtering [3], data aggregation[4] and transmission of information.

\subsection{Data Filtering}

Middleware receives massive EPC (Electronic Product Code) data from RFID readers, there is too much redundant information, and there exists a lot misread information. Therefore, it needs to filter the data and eliminate redundant data, and filter out unwanted information as well as send the useful information to the application. Redundant data includes (1) in the short term the same reader repeat to report the same data. (2) More than one near reader may report the same data in the different time.

The solution for redundancy data is to set the various filter processing. The typical filter has four categories: product filter, time filter, EPC filters and smoothing filter. Product filter transmits only the product information related to a product or the manufacturer, that is, the filter transmits only a certain range of EPC data. Time filter can filter events based on time records, for example, a time filter may only send events that happened within the last 10 minutes. EPC code filter can send only to meet certain rules EPC code. Smoothing filter can handle those cases of error, including leakage read and misread. According to actual needs, the filter can be like assembling toys, like stitching together one by one, in order to obtain the desired event. For example, a smoothing filter can combine with a product filter. It leads events to separate to an application.

\subsection{Data Aggregation}

Because source RFID data stream received from the reader are simple single fragmented information, for giving applications or other RFID middleware to provide meaningful information, it needs for RFID data aggregation processing. CEP (complex event processing) technology can be taken into consider. Complex event processing is an emerging technology, used to handle a large number of simple events and sort out valuable event. It can help people through the analysis of complex events, change the simple event into a valuable event and derive actionable information.

Through data aggregation, source RFID data is simplified into meaningful complex events, such as a RFID tag firstly emerged in the range of a reader detecting, then disappeared. By virtue of a number of simple data analysis, it can determine the tag into the event and leave the event. The aggregation can solve a problem caused by a temporary error reading data, making data smooth.

\subsection{Information Transmission}

After filtering and aggregation processing, RFID data need reach those entities interested in it, such as enterprise applications, information service system or other RFID middleware, that leads a messaging services mechanism to transmit RFID information.In the form of information-to-information, a message is transferred from one program to another program or programs. Information can transmit in an asynchronous manner, so the sender does not have to wait for a response. Message-oriented middleware functionality includes not only the transmission of information, but also include an explaining data, data security, data broadcasting, error recovery, locating network resources, computing the path cost, message priorities as well as excluding error and other services.

Under J2EE platform, JMS (Java Message Service[5]) can implement interacting messaging between RFID middleware and enterprise applications. Using JMS publish / subscribe model, RFID middleware publish a topic messages so that enterprise applications can order the subject of the message. Here the message is the specific language of things - PML (Physical Mark-up Language) format. Even though the database software to change information or increase RFID reader types, it does not need to modify the front-side application to perform data processing, eliminating the need for multi to multi complex maintenance.

\section{Layered Design}

The next discussion will start from functionality requirement, structural design of RFID middleware, and subsequently present an enterprise-level application solution based on RFID middleware.

\subsection{Functionality Analysis}

Required functionality: (1) RFID devices access: make the different RFID reader with RJ-45 Ethernet port, RS232 serial, USB and other interfaces access to the middleware system. (2) Data filtering: remove and filter the redundant data of RFID tags. (3) Reliable transmission of real-time data: requires the RFID terminal equipment to gather information in order to 
ensure a good communication between devices with the reliability of data source. (4) Software Human Interface: view real-time status of each RFID equipment running, also manage the tagged data, the interface should be simple and easy to operate.

\subsection{Middleware Hierarchy}

The design uses layered approach to achieve the above functions, the system is by the device management, data filtering layer and the service layer to handle different tasks, as shown in Fig. 1. Moreover, the various parts are as follows.

(1) Device management layer: create the physical connection between the main provider of middleware and RFID devices, and process the related services of monitoring equipment.

(2) Data filter layer: mainly filter raw RFID events, providing specific semantic information messages to the upper application systems.

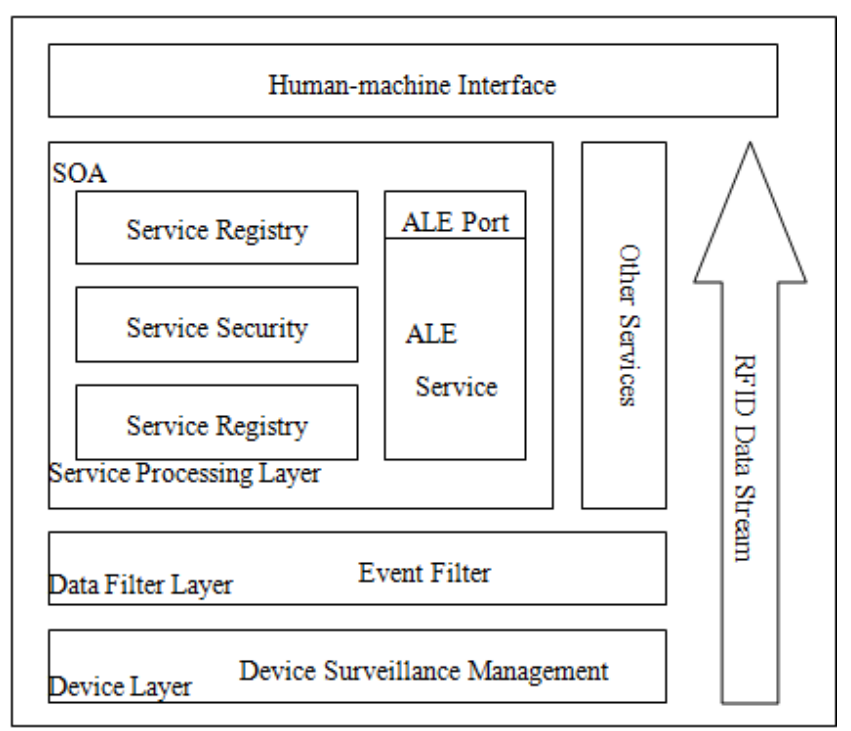

Figure 1. Middleware hierarchy

(3) Service processing layer: provide services to other applications through ALE (Application Level Events) interface. Service registration is used to define new services; security services provide secure transmission of messages; service management is to view a variety of services, reserved or revoked.

(4) Human-machine interface: real-time update RFID device information and achieve service inquiry.

(5) Other services: provide services for the enterprise ERP system and other management systems.

\section{Applied Model}

Based on the layered design idea, we construct a hierarchy structure of RFID middleware and present a kind of enterprise class applied model, in which RFID event manager module is responsible for processing data streams and RFID information service module is responsible for system integration.

\subsection{Application Framework}

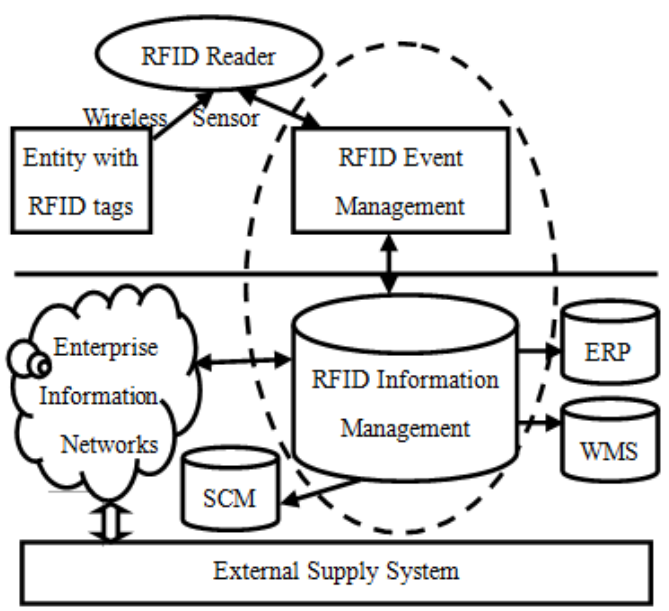

Figure 2. Application framework of RFID middleware

RFID middleware is essentially a service program that directly interacts with tag reader device. Its main functions include the RFID reader data extraction, filtering, processing and integration with other application interfaces, and the application framework is as shown in Figure 2.

In Figure2, the imaginary part of the coil is the RFID middleware. Reading continuously a large number of tags, the RFID reader transfers data to RFID middleware to process. Our design adopts SOA [6] (service-oriented architecture) architecture and divides the middleware into RFID event manager module and information-service module.

\subsection{RFID Event Manager Module}

This module is responsible for processing data streams from one or more RFID reader. Before data is sent to the relevant application, the data should be filtered and integrated pre-treatment. Therefore, it should set filtering mechanism in the module, discarding the redundant data that produces when the goods are not moving. The goods only when the state has changed, really triggers an action or event. The Event Manager module can also define additional filtering mechanism, through customized way to implement specific business logic, so that other software can continuously use relevant data.

RFID Event Manager Module involves: (1) device adapter sub-modules: use RFID air interface protocol to allow devices from different vendors can communicate and interact. (2) Filter sub-modules: customize semantic information and design of complex event processing mechanism to filter redundant data provided by RFID readers. (3) System connection: the underlying data can be sent to the file system, message queues and other places, to deliver RFID-related events and data to the upper application system.

\subsection{RFID Information Service Module}

The module connects to EIS (Enterprise Information System) which includes ERP (Enterprise Resource Plan), WMS (Warehouse Management System), SCM (Supply 
Chain Management) and other systems that hope to use the RFID tag information, as shown in Fig. 2 lower right. In fact, this module can be thought as the integration layer of enterprise-level application system, which provides a connection channel between RFID data base and high-layer business applications.

This module obtains data include: (1) the reader's type and other data. (2) The key data labelled by tags, such as weight, manufacturing date, the upstream enterprise and so on. The RFID information service module is set between the RFID event management module and other enterprise applications, so it can provide maximum flexibility according to the change of the business needs or enterprise applications, and reflect the superiority of SOA software architecture.

\section{Experiment Results}

In the detailed design stage, we select the java language and MS Access database as development tool, programming to implement RFID middleware for identifying the different hardware and redundant data filtering. The experiment uses an open-source software Rifidi [7] for simulation testing. The Rifidi can create a kind of software simulation environment of the RFID system. Its software toolkit can be based on the realities of RFID applications to simulate RFID reader/tag hardware's access and achieve the interaction between middleware and virtual devices.

\subsection{Simulation Testing}

Experimental scene simulates goods entering and leaving the warehouse. Goods are inspected at an entrance or outlet equipped with RFID reader. First, create two components in the simulation software: Dock Door-1 and DockDoor-2 (both with a certain type of reader), defines two-batch testing: The first batch test group has three tags; the second batch test group has four tags. Define entrance or exit two scenes, each with a defined reader. Run middleware and test RFID reader how to read batch tags. The experimental results test two tag group behaviors, that is, whether the first group behaviors match the scene 1 and batch 1 , and whether the second group match the scene 2 and batch 2 .

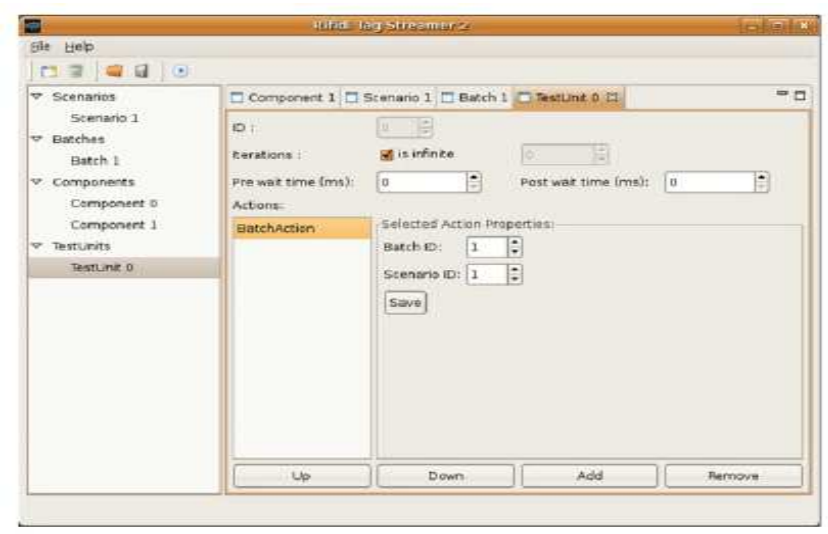

Figure 3. Simulation result
The console displays the matching results and the filtered tag data. After testing, successful identification-rate of RFID middleware reaches $100 \%$ and according to the results of the console displays, data-filtering module effectively filters many redundant data in tags. The simulation test interface is shown in Figure 3.

\subsection{Practical Testing}

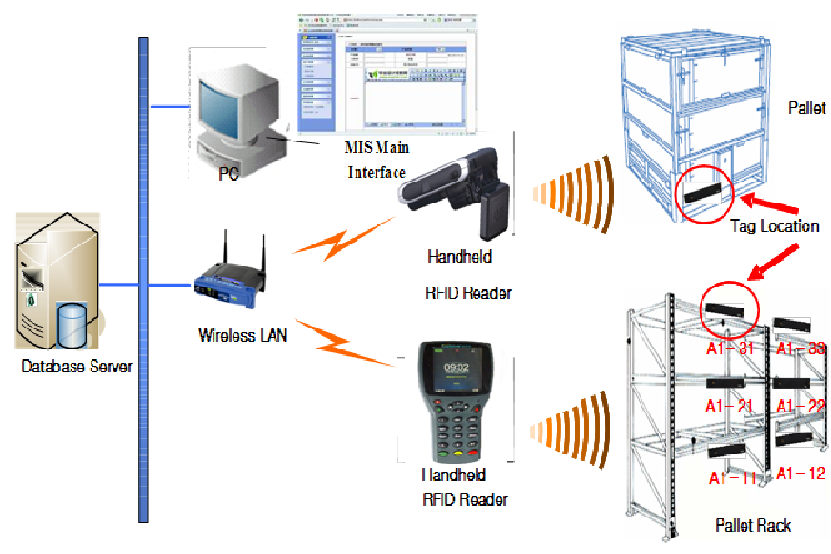

Figure 4. Detection to goods out of storage.

We apply our RFID middleware system to a logistics enterprise for detecting and tracking the goods out of storage, shown in Figure 4.

Practical results show that our system can display the physical location of goods in real time and enable redundant RFID data filtering and automatic data transformation. All the information on the items can be collected and tracked in real time, and provide a strong support to goods out of storage for its effective management and control.

\section{Conclusions}

The simulation and practical tests prove our RFID middleware system whose recognition rate, redundant data filtering capability and other performance indicators have reached the preset requirements. Moreover, device management layer can configure, operate and monitor the device; data management layer can process data and filtering tag data redundancy well; Event management can convert raw tag data into business events; application software can put easily the system at all levels into practice through application program interface.

From the application level, on this basis of the applied model of RFID middle system based on Internet of things, people can further research to establish general information processing custom rules, and enhance constructing efficiency. This is certainly conducive to facilitate practical application for Internet of things technology.

\section{Acknowledgements}

The research was supported by Artificial Intelligence Key Laboratory of Sichuan Province (No. 2013RYY04) and the 
Sichuan Provincial Education Department's Key Project (No.14ZA0210).

Our work was also supported by university Key Laboratory of Sichuan Province (No. 2013WYY09) and Fund Project of Sichuan Provincial Academician (Experts) Workstation (No.2014YSGZZ02).

\section{References}

[1] Hoag J E, Thompson C W. Architecting RFID middleware[J]. Internet Computing, IEEE, 2006, 10(5): 88-92.

[2] Kim G, Ryu W, Hong B, et al. Real-Time Quantity Inspection Method for Moving Tags in RFID Middleware[M]//Secure and Trust Computing, Data Management, and Applications. Springer Berlin Heidelberg, 2011: 77-83.

[3] Zhang M Z,et al. Research of data filtering model in embedded RFID middleware. Computer Engineering and Design, 31(17), pp. 3743-3746,2011.
[4] Tan H O, Korpeoglu I, Stojmenovic I, Computing localized power-efficient data aggregation trees for sensor networks. Parallel and Distributed Systems, IEEE Transactions on, 22(3), pp.489-500, 2011.

[5] Kuehnhausen M, Frost V S, Application of the Java Message Service in mobile monitoring environments. Journal of Network and Computer Applications, 34(5), pp. 1707-1716, 2011.

[6] Seinturier Lionel, et al. A component - based middleware platform for reconfigurable service - oriented architectures. Software: Practice and Experience, 42(5), pp. 559-583, 2012.

[7] Huebner A, Facchi C, Janicke H. Rifidi Toolkit: Virtuality for Testing RFID[C]//ICSNC 2012, The Seventh International Conference on Systems and Networks Communications. 2012: $1-6$. 